\title{
THE PENETRATION OF A FINGER INTO A VISCOUS FLUID IN A CHANNEL AND TUBE*
}

\author{
D. A. REINELT $\dagger$ AND P. G. SAFFMAN $\dagger$
}

\begin{abstract}
The steady-state shape of a finger penetrating into a region filled with a viscous fluid is examined. The two-dimensional and axisymmetric problems are solved using Stokes equations for low Reynolds number flow. To solve the equations, an assumption for the shape of the finger is made and the normal-stress boundary condition is dropped. The remaining equations are solved numerically by covering the domain with a composite mesh composed of a curvilinear grid which follows the curved interface, and a rectilinear grid parallel to the straight boundaries. The shape of the finger is then altered to satisfy the normal-stress boundary condition by using a nonlinear least squares iteration method. The results are compared with the singular perturbation solution of Bretherton (J. Fluid Mech., 10 (1961), pp. 166-188). When the axisymmetric finger moves through a tube, a fraction $m$ of the viscous fluid is left behind on the walls of the tube. The fraction $m$ was measured experimentally by Taylor (J. Fluid Mech., 10 (1961), pp. 161-165) as a function of the dimensionless parameter $\mu U / T$. The numerical results are compared with the experimental results of Taylor.
\end{abstract}

Key words. viscous fluid, fingering, singular perturbation, composite mesh

1. Introduction. We consider the penetration of a finger into a region which is initially filled with a viscous fluid. It is assumed that the viscosity of the fluid inside the finger is negligible when compared with the viscosity of the fluid exterior to the finger. The more general case, where the viscosity of the fluid inside is not neglected, can also be solved with the methods described below but is left for further study. The free boundary value problem for the steady-state shape of the finger is examined with two different geometries: the two-dimensional case of a finger between parallel plates and the axisymmetric case of a finger in a tube. It will be supposed that the gravitational and inertial forces are small in comparison with the viscous forces, and can be neglected. There is, in principle, no difficulty in incorporating their effect into the fingering problem with the present method.

The two-dimensional case is important in the study of fingering in a Hele-Shaw cell composed of two closely spaced parallel plates separated by a distance $2 b$. The sides of the cell connecting the two plates are a distance $2 l$ apart, where $l \gg b$. A finger, shaped like a tongue, moves through the Hele-Shaw cell with constant velocity $U$. The thickness of the tongue is $2 \beta b$ and its width is $2 \lambda l$, where the parameter $\beta$ is equal to (thickness of finger)/(distance between plates) and the parameter $\lambda$ is equal to (width of finger)/(width of cell). The determination of the value of $\lambda$ has been a subject of much interest. Experiments examining the shape of a finger in a Hele-Shaw cell have been performed by Saffman and Taylor (1958) and Pitts (1980). Since the full threedimensional problem is difficult to calculate, the problem of finding the shape of the finger in the plane parallel to the plates was approximated by averaging the velocity field across the gap between the two plates. This leads to two-dimensional equations in which the components of the mean velocity in the plane parallel to the plates are given by

$$
u=-\frac{b^{2}}{3 \mu} \frac{\partial p}{\partial x}, \quad w=-\frac{b^{2}}{3 \mu} \frac{\partial p}{\partial z}
$$

\footnotetext{
* Received by the editors September 13, 1983, and in revised form January 13, 1984. This work was supported by the U.S. Department of Energy, Office of Basic Energy Sciences, and the Office of Naval Research.

† Department of Applied Mathematics, California Institute of Technology, Pasadena, California 91125.
} 
where $\mu$ is the viscosity of the fluid and $p$ is the pressure in the fluid which is to this approximation a function only of $x$ and $z$. The plates are taken parallel to the $(x, z)$-plane, and the $y$-axis is normal to the plates with origin in the mid plane. The continuity equation

$$
\frac{\partial u}{\partial x}+\frac{\partial w}{\partial z}=0
$$

must also be satisfied. These equations hold in the region of the $(x, z)$-plane that is not occupied by the finger.

In the region of the $(x, z)$-plane where the finger is found, the approximate equations were given by Saffman (1982). In this region, there is on the surface of each plate a film of viscous fluid of total thickness $m b$, where $m \rightarrow 1-\beta$ away from the edge of the finger, in which the pressure is $p_{i}$ and the components of mean velocity are

$$
u_{i}=-\frac{m^{2} b^{2}}{3 \mu} \frac{\partial p_{i}}{\partial x}, \quad w_{i}=-\frac{m^{2} b^{2}}{3 \mu} \frac{\partial p_{i}}{\partial z} .
$$

The continuity equation for the viscous fluid is

$$
\frac{\partial m}{\partial t}+\frac{\partial\left(m u_{i}\right)}{\partial x}+\frac{\partial\left(m w_{i}\right)}{\partial z}=0
$$

The remaining equation is

$$
p_{i}+b T \nabla^{2} m=p_{0}
$$

where $T$ is the surface tension and $p_{0}$ is the constant pressure inside the finger, i.e. for $|y|<(1-m) b$.

The two-dimensional solutions in the two regions are joined by boundary conditions at the edge of the finger. First, there is the kinematic condition as the boundary of the finger is approached;

$$
(\mathbf{U}-\mathbf{u}) \cdot \mathbf{n}=m\left(\mathbf{U}-\mathbf{u}_{i}\right) \cdot \mathbf{n}
$$

where $\mathbf{U}=(U, 0)$ is the velocity of the finger, $\mathbf{u}=(u, w)$, and $\mathbf{n}=\left(n_{x}, n_{z}\right)$ is the normal to the edge of the finger. Second, there is a dynamic condition relating the limits of the pressure on the two sides,

$$
p-p_{i}=\Delta p \text {. }
$$

Here, the limits are to be understood as outer limits in which the distance from the edge of the finger is small compared with $l$, but large compared with $b$. The limiting values of $m$ and the pressure jump $\Delta p$ are so far unknown, but under the postulated conditions, we expect them to have the form

$$
m=F\left(\frac{\mu \mathbf{U} \cdot \mathbf{n}}{T}, \frac{b}{R}\right)
$$

and

$$
\Delta p=\frac{T}{b} f\left(\frac{\mu \mathbf{U} \cdot \mathbf{n}}{T}, \frac{b}{R}\right)
$$

where $R$ is the radius of curvature in the plane parallel to the plates. The functions $F$ and $f$ are to be determined by local (inner) solutions of the equations in the vicinity of the finger edge which take into account the $y$-dependence of the flow field and 
shape of the interface. As such solutions have not been available, it has been the practice to approximate them by the relations

$$
F=F_{0}, \quad f=f_{0}+f_{1} \frac{b}{R}
$$

where $F_{0}, f_{0}$, and $f_{1}$ are constants.

Saffman and Taylor (1958) assumed further that the surface tension $T$ could be neglected $\left(f_{1}=0\right)$ and were able to derive a closed form solution. They also found that the difference between the shape of the finger determined from their closed form solution and the shape observed from the experimental results was considerable unless $\lambda$ is close to $\frac{1}{2}$, but the parameter $\lambda$ was not determined by their analysis. McLean and Saffman (1981) have taken into account the effect of the surface tension $T$ by setting $f_{1}=-1$ in their examination of the fingering problem. This removed the indeterminancy associated with $T=0$ and gave $\lambda$ as a function of $\mu U / T .^{1}$ The shape of the finger with a given value of $\lambda$ was found to be in close agreement with the shape given by experimental results with the same value of $\lambda$. However, a comparison between a plot of $\lambda$ versus $\mu U / T$ using these results and the same plot using the experimental results showed significant disagreement. Rough agreement would be obtained for $f_{1}$ approximately equal to $-\frac{1}{2}$.

In this paper, we calculate

$$
F\left(\frac{\mu U}{T}, 0\right) \text { and } f\left(\frac{\mu U}{T}, 0\right)
$$

for finite $\mu U / T$. To determine these functions, it is necessary to solve the Stokes equations in the plane perpendicular to the plates. Bretherton (1961) has determined $F$ and $f$ for $\mu U / T \ll 1(b / R=0)$ by perturbation methods, and recently Park and Homsy (1983) have determined the $b / R$ correction for $\mu U / T \ll 1$ and $0<b / R \ll 1$. The use of boundary conditions incorporating finite $\mu U / T$ effects and the $b / R$ dependence can possibly be used to bring the plot of $\lambda$ versus $\mu U / T$ into closer agreement with experiments, and also explain the observed stability of the fingers. The results of Romero (1982) who explored the dependence of solutions on an assumed dependence of $F$ and $f$ on $\mu U / T$ showed that the $b / R$ term is essential to remove the degeneracy of the $T=0$ closed form solution.

Besides the two-dimensional problem, we also solve the penetration of an axisymmetric finger into a viscous fluid in a tube. The diameter of the tube is $2 b$ and the diameter of the finger moving through the tube with constant velocity $U$ is $2 \beta b$. The parameter $\beta$ is equal to (diameter of finger)/(diameter of tube). This problem has been investigated experimentally by Taylor (1961) and Cox (1962). The numerical results are compared with the experimental results and the agreement is found to be remarkably good.

In order to determine the solution to the two-dimensional and axisymmetric problems for $\mu U / T$ equal to $O(1)$, the free boundary value problem is solved in two stages. First, we begin with an initial guess for the shape of the finger. This can be found by starting with a small value for the parameter $\mu U / T$ and using Bretherton's solution. Since we have assumed a shape for the finger, we are forced to drop one of the boundary conditions applied on the curved interface; the normal-stress boundary condition is dropped. A system of equations equivalent to the biharmonic equation

\footnotetext{
${ }^{1}$ But as later found by Romero (1982) and Vanden-Broeck (1983), there are in fact more than one value of $\lambda$ for each $\mu U / T$.
} 
must now be solved on a fixed domain. It is important to use a numerical method that not only gives accurate results in the interior of the domain but also gives accurate results on the curved interface. To accomplish this, we cover the domain with a composite mesh composed of a curvilinear grid which follows the curved interface, and a rectilinear grid which is parallel to the straight boundaries. These overlapping grids are stretched so that the number of grid points is greatest in regions where they are needed most. Interpolation equations are used to connect the two grids. Finite difference methods are used to calculate the numerical solution.

In the second stage, the shape of the finger is altered to satisfy the normal-stress boundary condition. The curved interface is expanded in terms of Chebyshev polynomials and the known asymptotic behavior of the finger as $x \rightarrow-\infty$. Using the solution calculated on the fixed domain, the expansion of the interface, and the normal-stress boundary condition, a new shape for the interface is determined by a nonlinear least squares iteration method. After several iterations, the normal-stress boundary condition is satisfied and we have a solution.

The use of a composite mesh to cover the domain was suggested by Prof. H. O. Kreiss. We considered the employment of boundary integral methods and finite element techniques, but found them less convenient and they did not appear to offer improved accuracy or cheaper computations. The finite element method would have required using higher order elements with one curved side to conform to the interface. Both the composite mesh discussed above and a finite element mesh must be altered each time the interface changes. This was accomplished easily and with a small amount of computation time using the composite mesh technique. Also, the present method allows easy incorporation of inertial and nonuniform fluid effects; this is not the case for the boundary integral method.

2. Formulation of the two-dimensional problem. We examine the penetration of a finger of fluid into the narrow region between two closely spaced parallel plates. As mentioned earlier, it is assumed that the viscosity of the fluid inside the finger is negligible when compared with the viscosity of the fluid exterior to the finger. This allows us to solve the equations only in the region exterior to the finger. The steady state problem is examined where the finger is moving with constant velocity $U$ and is symmetrical about the center line of the channel. The plates are separated by a distance $2 b$ and the finger has asymptotic width $2 \beta b$.

The Stokes equations for incompressible two-dimensional low Reynolds number flow are

$$
\begin{aligned}
& \hat{u}_{\hat{x}}+\hat{v}_{\hat{y}}=0, \\
& \hat{p}_{\hat{x}}=\mu\left(\hat{u}_{\hat{x} \hat{x}}+\hat{u}_{\hat{y} \hat{y}}\right), \\
& \hat{p}_{\hat{y}}=\mu\left(\hat{v}_{\hat{x} \hat{x}}+\hat{v}_{\hat{y} \hat{y}}\right),
\end{aligned}
$$

where $\hat{p}$ is the pressure and $\mu$ is the viscosity of the fluid. The velocities $\hat{u}$ and $\hat{v}$ of the fluid are in the $\hat{x}$ and $\hat{y}$ direction respectively. The $\hat{y}$-axis is taken normal to the plates with origin in the mid plane. The tip of the finger moves along the $\hat{x}$-axis. Boundary conditions are applied on the plates $\hat{y}= \pm b$ and on the interface between the two fluids.

We now change to a reference frame moving with the finger. The tip of the finger is fixed at the origin. In this new reference frame, the velocities are independent of 
time. Dimensionless variables are introduced by

$$
\begin{gathered}
x=\frac{\hat{x}-U t}{b}, \quad y=\frac{\hat{y}}{b}, \quad R=\frac{\hat{R}}{b}, \\
u=\frac{\hat{u}-U}{U}, \quad v=\frac{\hat{v}}{U}, \quad p=\frac{\hat{p}}{T / b},
\end{gathered}
$$

where $R$ is the radius of curvature and $T$ is the surface tension. In the perturbation analysis of the fingering problem, it becomes clear that the appropriate scaling for $\hat{p}$ is $T / b$ and not $\mu U / b$. The use of the second scaling results in $p \rightarrow-\infty$ as $\mu U / T \rightarrow 0$. We substitute these new variables into (12) to get

$$
\begin{aligned}
& u_{x}+v_{y}=0, \\
& p_{x}=\mathrm{Ca}\left(u_{x x}+u_{y y}\right), \\
& p_{y}=\mathrm{Ca}\left(v_{x x}+v_{y y}\right),
\end{aligned}
$$

where $\mathrm{Ca}=\mu U / T$. The capillary number $\mathrm{Ca}$ is the ratio of the viscous force to the force of surface tension.

In solving the fingering problem numerically, it is convenient to express the equations in terms of the stream function and the vorticity. We substitute the stream function $\psi$ defined by

$$
u=\psi_{y}, \quad v=-\psi_{x},
$$

and the vorticity $\omega$ defined by

$$
\omega=v_{x}-u_{y}
$$

into (13). If the pressure is eliminated from the equations, we obtain

$$
\begin{aligned}
& \psi_{x x}+\psi_{y y}=-\omega, \\
& \omega_{x x}+\omega_{y y}=0 .
\end{aligned}
$$

On the interface, it is convenient to use an arc-length coordinate $s$ equal to zero at the origin and increasing along the curved interface. Using the arc-length coordinate, the tangent vector $\mathbf{t}$ is equal to $\left(x_{s}, y_{s}\right)$, and the normal vector $\mathbf{n}$, pointing into the finger, is equal to $\left(-y_{s}, x_{s}\right)$. The interface conditions are

$$
\begin{aligned}
& x_{s} \psi_{x}+y_{s} \psi_{y}=0, \\
& \left(y_{s}^{2}-x_{s}^{2}\right)\left(\psi_{y y}-\psi_{x x}\right)+4 x_{s} y_{s} \psi_{x y}=0, \\
& p-2 \mathrm{Ca}\left[\left(y_{s}^{2}-x_{s}^{2}\right) \psi_{x y}-x_{s} y_{s}\left(\psi_{y y}-\psi_{x x}\right)\right]=p_{0}-\frac{1}{R},
\end{aligned}
$$

where

$$
\frac{1}{R}=x_{s} y_{s s}-y_{s} x_{s s}
$$

Since the pressure can only be determined up to a constant, we are free to set the constant pressure $p_{0}$ inside the finger equal to zero. These three interface conditions can be rewritten as

$$
\begin{aligned}
& \psi=0 \\
& \omega-2 x_{s s} \psi_{x}-2 y_{s s} \psi_{y}=0 \\
& p-2 \mathrm{Ca}\left[\left(y_{s}^{2}-x_{s}^{2}\right) \psi_{x y}-x_{s} y_{s}\left(\psi_{y y}-\psi_{x x}\right)\right]+x_{s} y_{s s}-y_{s} x_{s s}=0 .
\end{aligned}
$$


The boundary condition (16b) is found by differentiating (15a) with respect to $s$ and using this equation to eliminate the $\psi_{x y}$ term in (15b).

It is assumed that the shape of the finger is symmetric in the $y$ direction; it is then only necessary to solve (14) for $y \geqq 0$. The symmetry conditions for $x \geqq 0$ are

$$
\psi(x, 0)=0, \quad \omega(x, 0)=0 .
$$

In the new reference frame, the no-slip condition on the wall becomes

$$
\psi(x, 1)=-(1-\beta), \quad \psi_{y}(x, 1)=-1 .
$$

As $x \rightarrow-\infty$, the width of the finger approaches a constant; thus, we get a constant velocity between the finger and the solid boundary. Poiseuille flow develops as $x \rightarrow \infty$. The asymptotic behaviors are

$$
\begin{aligned}
& \psi \rightarrow-y+\beta \text { and } \omega \rightarrow 0 \text { as } x \rightarrow-\infty \\
& \psi \rightarrow \frac{3}{2} \beta\left[y-\frac{1}{3} y^{3}\right]-y \text { and } \omega \rightarrow 3 \beta y \quad \text { as } x \rightarrow \infty .
\end{aligned}
$$

3. Asymptotic properties of the solution. The shape of a finger penetrating into a viscous fluid can be determined by using singular perturbation methods for small $\mathrm{Ca}$. This work is described in Reinelt (1983). It is an extension of the work of Bretherton (1961) and his analysis of the motion of long bubbles in tubes. The work differs from Bretherton's work in that it outlines a procedure to develop a complete asymptotic expansion in terms of $\mathrm{Ca}$. It also constructs the equations in the boundary layer region in terms of scaled coordinates of order unity. The method of matched asymptotic expansions is used to connect the inner and outer solutions.

From Bretherton's solution or the solution using singular perturbation methods, $\beta$ is given by

$$
\beta \sim 1.0-1.337 \mathrm{Ca}^{2 / 3} .
$$

This expression, valid for small values of $\mathrm{Ca}$, holds for both the two-dimensional and axisymmetric problems. It will be compared with the numerical results.

The asymptotic behavior of the solution as $x \rightarrow-\infty$ can be expanded in powers of $\exp (k x)$ for finite values of $\mathrm{Ca}$. This leads to a relationship between $\mathrm{Ca}, \beta$, and $k$, the decay rate as $x \rightarrow-\infty$. The relationship will also be used to check the numerical results.

For the two-dimensional solution, the stream function takes the form

$$
\psi(x, y) \sim-y+\beta+e^{k x} g(y)+O\left(e^{2 k x}\right) .
$$

We substitute this expression into (14) to get an equation for $g(y)$,

$$
g_{y y y y}+2 k^{2} g_{y y}+k^{4} g=0 .
$$

The solution to this equation is a combination of the functions $\sin k y, \cos k y, y \sin k y$, and $y \cos k y$. If we satisfy the boundary conditions on the wall (18), we get the following expressions for the stream function, vorticity, pressure, and the shape of the interface:

$$
\begin{aligned}
& \psi(x, y) \sim-y+\beta+e^{k x}[A[k(y-1) \cos k(y-1)-\sin k(y-1)]+B k(y-1) \sin k(y-1)], \\
& \omega(x, y) \sim 2 k^{2} e^{k x}[A \sin k(y-1)-B \cos k(y-1)], \\
& p(x, y) \sim-2 \mathrm{Ca} k^{2} e^{k x}[A \cos k(y-1)+B \sin k(y-1)], \\
& y(x) \sim \beta-D e^{k x}
\end{aligned}
$$


where $A, B$, and $D$ are unknown constants. The above expressions are substituted into the three interface conditions $(16 \mathrm{a}, \mathrm{b}, \mathrm{c})$. If we keep only terms of $O(\exp k x)$, then the three equations for $A, B$, and $D$ are given by the matrix equation

$$
\left[\begin{array}{ccc}
-q \cos q+\sin q & q \sin q & 1 \\
q \sin q & \cos q & 1 \\
-2 \mathrm{Ca}(\cos q+q \sin q) & -2 \mathrm{Ca} q \cos q & 1
\end{array}\right]\left[\begin{array}{l}
A \\
B \\
D
\end{array}\right]=\left[\begin{array}{l}
0 \\
0 \\
0
\end{array}\right]
$$

where $q=k(1-\beta)$. The determinant of the matrix must be set equal to zero for a solution other than the trivial solution. This leads to an equation for $q$ in terms of $\mathrm{Ca}$,

$$
2 q-\sin 2 q+\mathrm{Ca}\left(4 q^{2}-4 \cos ^{2} q\right)=0 \text {. }
$$

The leading order expansion of (20) as $\mathrm{Ca} \rightarrow 0$ gives

$$
q \sim(3 \mathrm{Ca})^{1 / 3}
$$

which agrees with the singular perturbation solution. The relationships (20) and (21) between $k(1-\beta)$ and $\mathrm{Ca}$ will be compared with the numerical results.

A similar procedure was applied to the axisymmetric problem by Cox (1962) which led to an equation involving the three parameters $\mathrm{Ca}, \beta$, and $k$. In the experiments, the value of $k$ was determined by fitting the finger profile with an exponential curve. In the numerical treatment of the problem, $k$ is one of the parameters used to describe the interface; its value will be determined by satisfying the equations and boundary conditions.

4. Numerical solution on a fixed domain. To solve the fingering problem numerically, we begin with an initial guess for the shape of the finger. The initial guess is found by starting with a small value for $\mathrm{Ca}$ and using the perturbation solution. Since we have assumed a shape for the finger, we are forced to drop one of the three interface conditions $(16 \mathrm{a}, \mathrm{b}, \mathrm{c})$; the normal-stress boundary condition $(16 \mathrm{c})$ is dropped. The shape of the finger will be altered to satisfy this condition.

It is important to develop numerical methods that not only give accurate results in the interior of the region, but also give accurate results at the boundaries. To satisfy the normal-stress boundary condition, it is necessary to compute the pressure and the stresses accurately on the boundary. To accomplish this, we cover the domain with a composite mesh composed of a curvilinear grid which follows the curved interface, and a rectilinear grid which is parallel to the straight boundaries. Kreiss (1983) has developed a numerical code that constructs a curvilinear grid using spline interpolation that follows the smooth boundary of a simply connected domain. The rest of the domain is covered with a uniformly spaced rectilinear grid. The overlapping grids are used to solve a system of hyperbolic differential equations. We have modified these methods to treat the elliptic problem in this paper.

In the numerical treatment of the fingering problem, we restrict the infinite domain given by $-\infty<x<\infty$ and $0 \leqq y \leqq 1$ to a finite domain given by $x_{\min } \leqq x \leqq x_{\max }$ and $0 \leqq y \leqq 1$. If the values of $x_{\min }$ and $x_{\max }$ have been chosen properly, the difference between the numerical solution calculated on this domain and the solution calculated on an even larger domain will be small. As mentioned in $\S 2$, the domain is further restricted to the region exterior to the finger.

In the fingering problem, stretching is used in the curvilinear grid to place more grid points at the tip of the finger and fewer grid points where the width of the finger approaches a constant. To construct the curvilinear grid, we begin with a square grid 
with uniformly distributed grid points given by

$$
\left(\hat{s}_{i}, \hat{r}_{j}\right)=(i-1 / N-1, j-1 / M-1), \quad \text { where } i=1,2, \cdots, N \text { and } j=1,2, \cdots, M \text {. }
$$

There are $N$ grid points in the $\hat{s}$ direction and $M$ grid points in the $\hat{r}$ direction. The curvilinear grid is defined by mapping this square grid onto a region which follows the curved interface using a transformation $T_{c}$. To simplify the interface conditions, it is convenient to use the arclength parameter $s$ along the interface. Stretching is introduced by the transformations

$$
\hat{s}=F(s), \quad \hat{r}=G(r),
$$

where $F$ and $G$ are functions that produce a one-to-one mapping between the two sets of variables. The functions $F$ and $G$ are given in the appendix.

To construct the transformation $T_{c}$, cubic spline interpolation is used to approximate the shape of the curved interface through the $N$ grid points on the interface of the finger. The boundary $\hat{r}=0$ of the square grid is mapped onto the interface curve $C_{1}$ by

$$
x(s, 0)=X_{1}(s), \quad y(s, 0)=Y_{1}(s),
$$

where $X_{1}$ and $Y_{1}$ are cubic spline functions. Another set of $N$ points is chosen on a curve that lies in the interior of the domain under consideration. The interior curve used is a modified version of the Saffman-Taylor solution

$$
\exp \left[k_{1}\left(x-x_{1}\right)\right]=\cos \left[\frac{\pi y}{2 \beta_{1}}\right]
$$

where $k_{1}, x_{1}$, and $\beta_{1}$ are chosen constants. The transformation of the boundary $\hat{r}=1$ onto the interior curve $C_{M}$ is also done by cubic spline interpolation and given by

$$
x(s, 1)=X_{M}(s), \quad y(s, 1)=Y_{M}(s) .
$$

The curves, $C_{1}$ and $C_{M}$, form the two curved boundaries of the curvilinear grid. The corresponding grid points on these two curves are connected by straight lines. The complete transformation $T_{c}$ is

$$
\begin{aligned}
& x(s, r)=(1-r) X_{1}(s)+r X_{M}(s), \\
& y(s, r)=(1-r) Y_{1}(s)+r Y_{M}(s),
\end{aligned}
$$

with

$$
s=F^{-1}(\hat{s}), \quad r=G^{-1}(\hat{r}) .
$$

The function $G$ is chosen such that $G(1)=1$. This transformation is one-to-one and its Jacobian is never singular. A typical curvilinear grid is shown in Fig. 1.

Stretching is also used in the rectilinear grid to place a smaller mesh size near $y=1$ where the fluid moves into the narrow region between the finger and the wall. In the $x$ direction, we place fewer grid points near the boundaries at $x_{\min }$ and $x_{\max }$. To construct the transformation $T_{r}$, we begin with another square grid with uniformly distributed grid points given by

$$
\left(\hat{x}_{i}, \hat{y}_{j}\right)=\left(\frac{i-1}{N_{x}-1}, \frac{j-1}{N_{y}-1}\right), \quad \text { where } i=1,2, \cdots, N_{x} \text { and } j=1,2, \cdots, N_{y} .
$$

The number of grid points in $\hat{x}$ and $\hat{y}$ directions are $N_{x}$ and $N_{y}$ respectively. The 


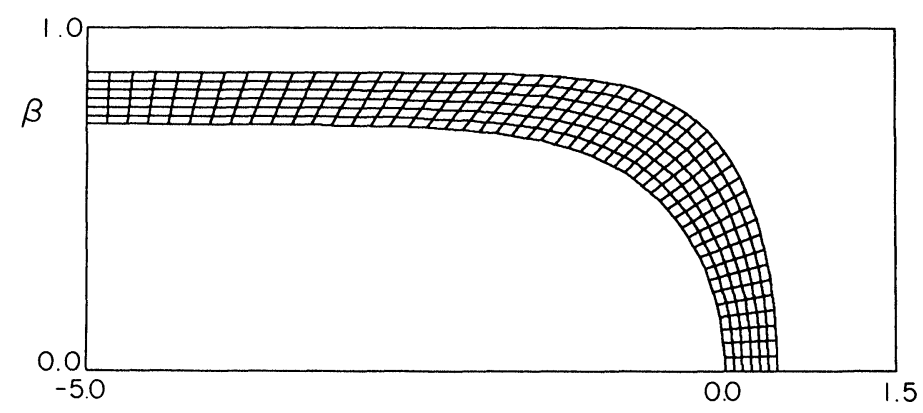

FIG. 1. Typical curvilinear grid.

transformation $T_{r}$ given by

$$
\hat{x}=f(x), \quad \hat{y}=g(y),
$$

maps the square grid onto the rectilinear grid. The functions $f$ and $g$ are given in the appendix.

Many of the grid points in the rectilinear grid are in the interior of the finger. These points are not used in the computation of the solution. Figure 2 gives an example of a rectilinear grid that shows only the grid points actually used. It is important that the grids overlap so that all grid points on $C_{M}$ lie in the interior of the rectilinear grid. Also, the grid points on the jagged boundary of the rectilinear grid must lie in the interior of the curvilinear grid.

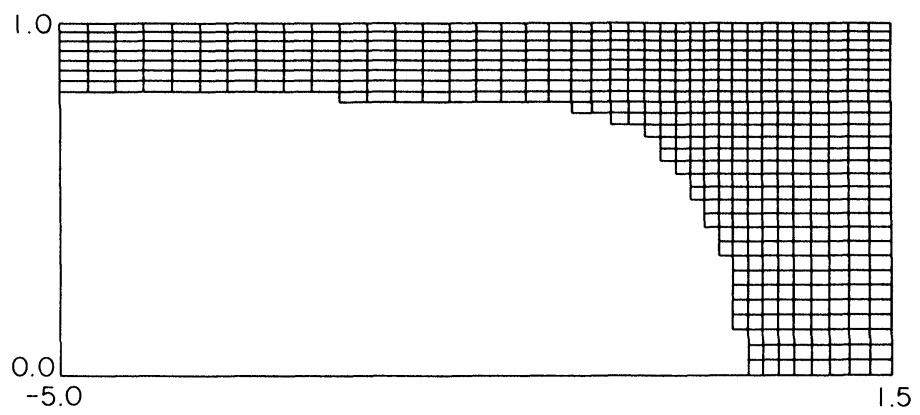

FIG. 2. Typical rectilinear grid.

In solving the equations on a composite mesh, the grid points can be divided into three categories. At interior points of each grid, difference equations that approximate the partial differential equations are applied. At grid points that lie on the boundary of the domain, boundary conditions are applied. The third type of grid points are those that lie on the interior curve $C_{M}$ of the curvilinear grid and those that lie on the jagged boundary of the rectilinear grid. It is at these grid points that interpolation equations are used to connect the solutions on the two grids.

At interior points of each grid, the system of equations (14) is replaced by difference equations at the uniformly distributed grid points of the two square grids. These grids are related to the rectilinear and curvilinear grids through the transformations $T_{r}$ and $T_{c}$. For example, the stream function equation (14a) is written in terms of $\hat{x}$ and $\hat{y}$ coordinates by using (24),

$$
f^{\prime}(x) \frac{\partial}{\partial \hat{x}}\left[f^{\prime}(x) \frac{\partial \psi}{\partial \hat{x}}\right]+g^{\prime}(y) \frac{\partial}{\partial \hat{y}}\left[g^{\prime}(y) \frac{\partial \psi}{\partial \hat{y}}\right]=-\omega
$$


where

$$
x=f^{-1}(\hat{x}), \quad y=g^{-1}(\hat{y}) .
$$

Using the notation $\psi_{i, j}=\psi\left(\hat{x}_{i}, \hat{y}_{j}\right)$, a difference equation for (25) is given by

$$
\begin{aligned}
& \frac{f_{i}^{\prime}}{h_{x}}\left[f_{i+1 / 2}^{\prime}\left(\frac{\psi_{i+1, j}-\psi_{i, j}}{h_{x}}\right)-f_{i-1 / 2}^{\prime}\left(\frac{\psi_{i, j}-\psi_{i-1, j}}{h_{x}}\right)\right] \\
& +\frac{g_{j}^{\prime}}{h_{y}}\left[g_{j+1 / 2}^{\prime}\left(\frac{\psi_{i, j+1}-\psi_{i, j}}{h_{y}}\right)-g_{j-1 / 2}^{\prime}\left(\frac{\psi_{i, j}-\psi_{i, j-1}}{h_{y}}\right)\right]=-\omega_{i, j}
\end{aligned}
$$

where the mesh sizes $h_{x}$ and $h_{y}$ are

$$
h_{x}=\frac{1}{N_{x}-1}, \quad h_{y}=\frac{1}{N_{y}-1} .
$$

The expression $f_{i+1 / 2}^{\prime}$ is defined by

$$
f_{i+1 / 2}^{\prime}=\frac{1}{2}\left[f^{\prime}\left(x_{i}\right)+f^{\prime}\left(x_{i+1}\right)\right] .
$$

This second order accurate difference equation is used at all interior grid points of the $(\hat{x}, \hat{y})$ square grid. A similar procedure is used to find the difference equations to be applied at the uniformly distributed grid points of the $(\hat{s}, \hat{r})$ square grid.

The computational boundary conditions for the fingering problem must be chosen carefully. One method of applying the boundary conditions at $y=1$ is to construct the grid with the boundary $y=1$ centered between the top two grid lines. This allows us to give the value of $\psi$ on the top two grid lines. However, this approach leads to an $O(1)$ error in the vorticity near the corner $x=x_{\max }$ and $y=1$. To avoid these problems, we construct the rectilinear grid with the top grid line coincident with $y=1$. A second order accurate equation for the vorticity on the boundary $y=1$ can now be written using $\psi$ on the top three grid lines and $\psi_{y}$ given on the boundary. A more complete discussion of the boundary conditions can be found in Reinelt (1983).

On the interface, the curvilinear grid is constructed with the grid line $(\hat{r}=0)$ coincident with the shape of the interface curve. The boundary conditions applied on the interface are written in terms of $s$ and $r$ coordinates by

$$
\psi=0, \quad \omega-2\left(x_{s s} r_{x}+y_{s s} r_{y}\right) \psi_{r}=0
$$

The value of $\psi_{r}$ is calculated to second order by using the first three grid lines in the $r$ direction.

The values of $\psi$ and $\omega$ at the third type of grid point are determined by interpolating between the two grids. A nine point formula and a four point formula were examined for this interpolation. The nine point formula was chosen because a test of calculating an exact solution with inhomogeneous boundary conditions showed that a four point formula was not accurate enough. The interpolation equations are discussed in terms of a smooth function $u$. To simplify the interpolation formulas, we use formulas based on the uniformly distributed grid points of the two square grids. Each grid point on the curve $C_{M}$, given by $\left(s_{i}, 1\right)$, can be located in the interior of the $(\hat{x}, \hat{y})$ square grid by using (22) and (24). If $\left(\hat{x}_{0}, \hat{y}_{0}\right)$ is the location of one of these grid points, then the approximate value of $u$ at this grid point can be found by using the nine point 
interpolation formula given by

$$
\begin{gathered}
u\left(\hat{x}_{0}, \hat{y}_{0}\right)=\sum_{i=1}^{3} \sum_{j=1}^{3} d_{i}(\alpha) d_{j}(\gamma) u\left(\hat{x}_{I+i-2}, \hat{y}_{J+j-2}\right), \\
d_{1}(\alpha)=-\frac{1}{2} \alpha(1-\alpha), \quad d_{2}(\alpha)=(1-\alpha)(1+\alpha), \quad d_{3}=\frac{1}{2} \alpha(1+\alpha), \\
\alpha=\frac{\hat{x}_{0}-\hat{x}_{I}}{\hat{x}_{I+1}-\hat{x}_{I}}, \quad \gamma=\frac{\hat{y}_{0}-\hat{y}_{J}}{\hat{y}_{J+1}-\hat{y}_{J}},
\end{gathered}
$$

where $\left(\hat{x}_{I}, \hat{y}_{J}\right)$ is the grid point closest to the point $\left(\hat{x}_{0}, \hat{y}_{0}\right)$.

To find the approximate value of $u$ at each $(x, y)$ grid point on the jagged boundary, we locate each of these grid points in the interior of the $(\hat{s}, \hat{r})$ square grid. These values are found by using Newton's method and (22) and (23). Once these points are located, the interpolation formulas are identical with (26) where $\hat{x}$ and $\hat{y}$ are replaced by $\hat{s}$ and $\hat{r}$.

5. Iteration method. To determine the degree to which the normal-stress boundary condition is satisfied, it is necessary to find the pressure and the stresses on the interface. The pressure is calculated from the vorticity solution by integrating along the interface. The pressure is given in terms of the vorticity by

$$
p_{x}=-\mathrm{Ca} \omega_{y}, \quad p_{y}=\mathrm{Ca} \omega_{x} .
$$

Using the transformation $T_{c}$ and these relationships between the pressure and the vorticity, the derivative of the pressure with respect to arc length is

$$
p_{s}=-\mathrm{Ca}\left[x_{s} r_{y}-y_{s} r_{x}\right] \omega_{r}-\mathrm{Ca}\left[x_{s} s_{y}-y_{s} s_{x}\right] \omega_{s} .
$$

The stresses $\psi_{x x}, \psi_{x y}$, and $\psi_{y y}$ are calculated at each grid point on the curved interface from the stream function and vorticity solutions. We substitute the initial guess for the shape of the interface and the values of the pressure and stresses at each grid point on the interface into the normal-stress boundary condition (16c). If this boundary condition is satisfied, we have determined the shape of the finger. Normally, the right-hand side of the normal-stress boundary condition is not equal to zero at each grid point, but a residual $R_{i}$ is present. These residuals $R_{i}, i=1,2, \cdots, N$ give the error in the boundary condition (16c) at each of the $N$ grid points along the interface of the finger. In our calculations the value of $N$ is seventy-six. The shape of the interface must now be changed until all the residuals are smaller than a chosen error tolerance.

To change the shape of the interface, it is convenient to expand the interface in terms of a set of functions and unknown parameters. The shape of the finger is determined by the numerical values of these parameters. The form of the expansion greatly affects the amount of computing time needed to converge to the interface shape that satisfies the normal-stress boundary condition. In fact, if the expansion is not chosen properly, the problem may never converge.

The interface is expanded as a function of $y$. The expansion for the shape of the finger is given by

$$
x(y)=\frac{1}{k} \log \left\{\left[1-\left(\frac{y}{\beta}\right)^{2}\right]\left[1+\left(\frac{y}{\beta}\right)^{2} \sum_{j=0}^{m} c_{j} T_{2 j}\left(\frac{y}{\beta}\right)\right]\right\}
$$

where $\beta, k, c_{0}, c_{1}, \cdots, c_{m}$ are the parameters that determine the shape of the interface. The expansion is constructed so that the tip of the finger is located at the origin and $x(-y)$ is equal to $x(y)$. The functions $T_{2 j}$ are the even Chebyshev polynomials. If the 
grid points on the interface are projected onto the $y$-axis, there are many more points near the ends of the interval, $-\beta \leqq y \leqq \beta$, than near the center of the interval. This is characteristic of the so-called Chebyshev abscissae. The Chebyshev polynomials are chosen because it is expected that they will converge rapidly given the distribution of grid points used in the fingering problem. This is indeed found to be the case.

The asymptotic behavior of the shape of the finger as $x \rightarrow-\infty$ is

$$
y \sim \beta-D \exp (k x) .
$$

This relationship is inverted to give

$$
x \sim \frac{1}{k} \log \left[\frac{\beta}{D}\left(1-\frac{y}{\beta}\right)\right]
$$

as $y \rightarrow \beta$. The expansion is constructed so that this asymptotic behavior is included. If this is a good expansion, the value of $c_{j}$ will decrease as $j$ increases. This allows us to use the finite series from $j=1$ to $m$ as a good approximation to the infinite series.

The problem is now reduced to finding the parameters $\beta, k, c_{0}, c_{1}, \cdots, c_{m}$ that satisfy the equations

$$
R_{i}\left(\beta, k, c_{0}, c_{1}, \cdots, c_{m}\right) \approx 0, \quad i=1,2, \cdots, N .
$$

This is an overdetermined nonlinear system of equations because the number of grid points $(N)$ is larger than the number of parameters $(m+3)$. These equations are solved by determining the parameters that minimize the function

$$
\varphi\left(\beta, k, c_{0}, c_{1}, \cdots, c_{m}\right)=R_{1}^{2}+R_{2}^{2}+\cdots+R_{N}^{2} .
$$

To do this, we linearize the equations (28a) about an initial set of parameters $\beta^{\nu}, k^{\nu}, c_{0}^{\nu}, c_{1}^{\nu}, \cdots, c_{m}^{\nu}$.

$$
R_{i}\left(\beta^{\nu}, k^{\nu}, c_{0}^{\nu}, c_{1}^{\nu}, \cdots, c_{m}^{\nu}\right)+\frac{\partial R_{i}}{\partial \beta}\left(\beta-\beta^{\nu}\right)+\cdots+\frac{\partial R_{i}}{\partial c_{m}}\left(c_{m}-c_{m}^{\nu}\right) \approx 0 .
$$

This leads to a matrix equation containing the $N \times(m+3)$ Jacobian of (28a). The new values of the parameters are now determined by the method of least squares. The process is repeated until the values of $R_{i}, i=1,2, \cdots, N$ are smaller than $1 \times 10^{-3}$.

In the above calculations there is not a simple functional relationship between $R_{i}$ and the unknown parameters because the values of the pressure $p$ and the stresses $\psi_{x x}$, $\psi_{x y}$, and $\psi_{y y}$ depend on the parameters in some unknown way. In order to calculate the Jacobian of (28a), a small step size $h$ is added to each parameter independently and the new values of $\boldsymbol{R}_{i}$ are determined. For example, we calculate

$$
R_{i}\left(\beta^{\nu}+h, k^{\nu}, c_{0}^{\nu}, c_{1}^{\nu}, \cdots, c_{m}^{\nu}\right)
$$

which is used to determine the entries of the Jacobian

$$
\frac{\partial R_{i}}{\partial \beta}=\frac{R_{i}\left(\beta^{\nu}+h, \cdots\right)-R_{i}\left(\beta^{\nu}, \cdots\right)}{h} .
$$

$R_{i}$ is calculated $m+3$ times, once for each of the parameters. Each time the interface changes a new curvilinear grid is constructed. The calculations needed to determine the new grid and the transformation $T_{c}$ are a very small portion of the total computing time. The major portion of the computing time is needed to determine the numerical values of $\psi$ and $\omega$ on each of the fixed domains. In calculating the entries of the Jacobian, we can greatly reduce this time by not solving the entire system of equations directly each time. 
On each of the fixed domains a large sparse system of linear equations for the values of $\psi$ and $\omega$ at the grid ponts of the rectilinear and curvilinear grids must be solved. If $\mathbf{v}$ is the vector that contains $\psi$ and $\omega$, then the system of linear equations can be written

$$
\mathbf{A v}=\mathbf{b}
$$

To solve this system of linear equations, we determine the $\mathbf{L U}$ decomposition of the matrix $\mathbf{A}$, where $\mathbf{L}$ is a lower triangular matrix and $\mathbf{U}$ is an upper triangular matrix. This linear system of equations now decomposes into two triangular systems that are solved by forward substitution and back-substitution. This decomposition of $\mathbf{A}$ involves a major portion of the computation time and is done using a sparse matrix solver (odrv, ndrv) developed at Yale University.

In order to calculate the values of $R_{i}\left(\beta^{\nu}+h, k^{\nu}, c_{0}^{\nu}, \cdots, c_{m}^{\nu}\right)$ and the values of the $R_{i}$ 's found by perturbing the other parameters, it is necessary to solve a new system of linear equations

$$
\overline{\mathbf{A}} \overline{\mathbf{v}}=\overline{\mathbf{b}} .
$$

Since this new system of equations is a perturbation of the original system of equations, it can be rewritten as

$$
\left(\mathbf{A}+\mathbf{A}_{1}\right) \overline{\mathbf{v}}=\left(\mathbf{b}+\mathbf{b}_{1}\right)
$$

where $\mathbf{A}$ and $\mathbf{b}$ are the matrices in the original system. The matrices $\mathbf{A}_{1}$ and $\mathbf{b}_{1}$ contain the small perturbations to the original system for small values of $h$. If we set

$$
\overline{\mathbf{v}}=\mathbf{v}+\mathbf{v}_{1}+\mathbf{v}_{2}+\mathbf{v}_{3}+\cdots
$$

then the solution to the new system of equations can be determined by solving the following equations:

$$
\begin{aligned}
& \mathbf{A} \mathbf{v}_{1}=\mathbf{b}_{1}-\mathbf{A}_{1} \mathbf{v}, \\
& \mathbf{A v}_{2}=-\mathbf{A}_{1} \mathbf{v}_{1} \\
& \mathbf{A} \mathbf{v}_{3}=-\mathbf{A}_{1} \mathbf{v}_{2}
\end{aligned}
$$

Since the $\mathbf{L U}$ decomposition of $\mathbf{A}$ is known and the right-hand side of each of these equations is known from the previous step, these equations are easily solved by forward substitution and back-substitution. In practice, the value of $\overline{\mathbf{v}}$ is determined to six places by solving only two or three of these equations. Using this method, the computation time necessary to compute the Jacobian is essentially equivalent to the time needed to solve the original system.

6. Numerical results for the two-dimensional problem. The numerical results are calculated by beginning with $\mu U / T=0.01$ and using the shape of the perturbation solution. Several iterations are needed to satisfy the normal-stress boundary condition. The value of $\mu U / T$ is then increased by small increments. The size of the increments varied from 0.02 for $\mu U / T<0.10$ to 0.20 for $\mu U / T>1.00$. The shape of the interface at the previous value of $\mu U / T$ is used as the basis for determining the new interface shape at the subsequent value of $\mu U / T$. Three or four iterations are needed for the normal-stress boundary condition to be satisfied at each value of $\mu U / T$ which corresponds to about 25 minutes of CPU on a VAX 11/750. 
The typical number of grid points used in each direction of the curvilinear and rectilinear grids is

$$
N=76, \quad M=7, \quad N_{x}=55, \quad N_{y}=34 .
$$

The value of $x_{\min }$ is determined by the choice of $s_{\max } ; \chi_{\min }$ is approximately equal to -5.0. The value of $x_{\max }$ is equal to 2.0. The shape of the finger is determined by using nine parameters $(m=6)$ for the expansion of the interface given in (27). The magnitude of the final coefficient $c_{6}$ is $O\left(10^{-4}\right)$. The inclusion of a greater number of parameters has very little effect on the shape of the finger.

Figure 3 is a plot of $q=k(1-\beta)$ versus $\mu U / T$. The solid line is a plot of (20) which was determined by expanding the solution in terms of eigenfunctions as $x \rightarrow-\infty$.

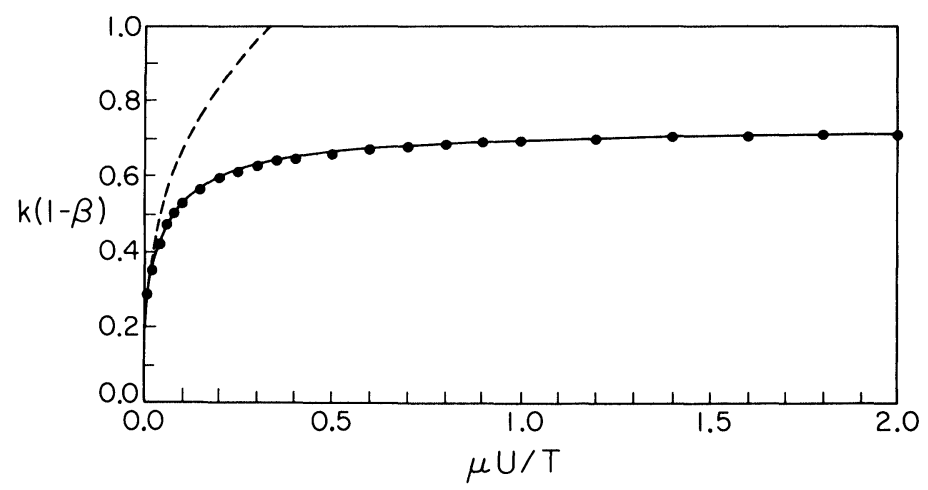

FIG. 3. The relationship $k(1-\beta)$ versus $\mu U / T$. $\bullet$, numerical results; _- plot of equation $(20) ;---$, perturbation result $(21)$.

The dots shown on the plot are the values of $k(1-\beta)$ calculated from the numerical results. The numerical results are in close agreement with the analytical result. The dashed line is a plot of the perturbation solution (21). As mentioned in $\S 3$, it is equivalent to the leading order behaviour of $(20)$. The perturbation solution (21) is in error by no more than $10 \%$ provided $\mu U / T<2 \times 10^{-2}$. In Fig. 4 , the solid line is a plot of $\beta$ versus $\mu U / T$ calculated from the numerical solutions and the dashed line is a plot of (19) determined from the perturbation solution. The function $F$ discussed

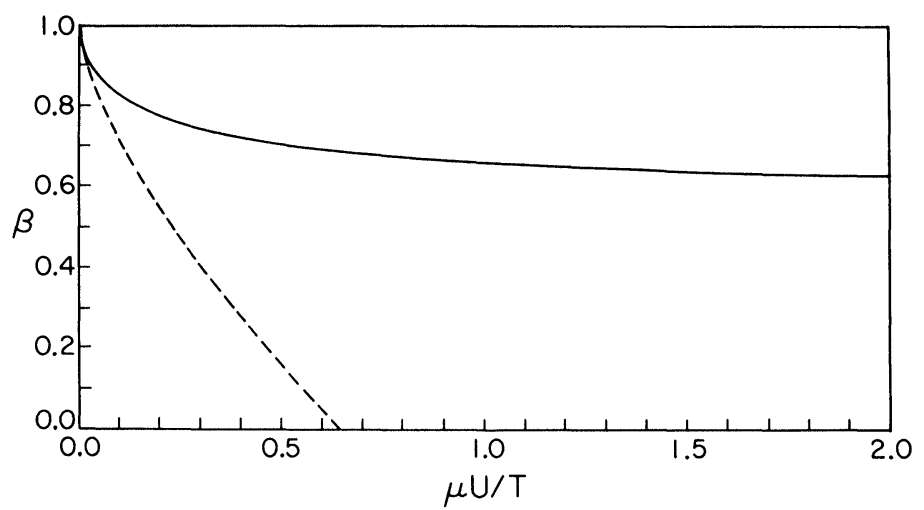

FIG. 4. The finger width $\beta$ versus $\mu U / T$ for the two-dimensional problem. _ _ numerical results; ---, perturbation result (19). 
in the introduction is given by

$$
1-\beta=F\left(\frac{\mu U}{T}, 0\right)
$$

As $x \rightarrow \infty$, the pressure is given by

$$
p \sim-3 \beta \mathrm{Ca} x+c_{p}
$$

where $c_{p}$ is a constant. It is this constant that gives the pressure jump that is needed in the two-dimensional approximation of the flow in the Hele-Shaw cell,

$$
c_{p}=f\left(\frac{\mu U}{T}, 0\right) \text {. }
$$

The dotted line in Fig. 5 is a plot of $c_{p}$ versus $\mu U / T$ and the solid line is the actual pressure drop $\Delta p$ across the tip of the finger. The dashed line is the pressure drop calculated by Bretherton (1961) for $\mathrm{Ca} \rightarrow 0$ and is given by

$$
\Delta p \sim-1.0-3.8 \mathrm{Ca}^{2 / 3} \text {. }
$$

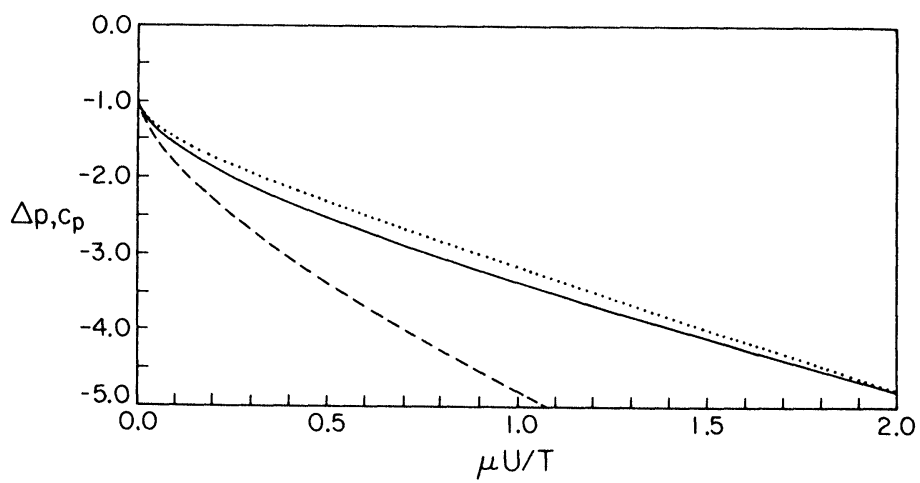

FIG. 5. Pressure drop $\Delta p$ across tip of the finger versus $\mu U / T$ for the two-dimensional problem. numerical results; ---, perturbation result; $\cdots, c_{p}$ versus $\mu U / T$.

This perturbation solution is equal to $c_{p}$ up to the order calculated. Both $c_{p}$ and $\Delta p$ have been normalized by $T / b$.

As $x \rightarrow \infty$, the velocity in the $x$-direction is

$$
u \rightarrow \frac{3}{2} \beta\left(1-y^{2}\right)-1 \text {. }
$$

When the value of $\beta$ is greater than $\frac{2}{3}$, the fluid near the $x$-axis moves with a velocity greater than that of the finger. In this case, two additional stagnation points are present on the interface. For all values of $\beta$, there is a stagnation point at the tip of the finger. Figure 6 gives examples of the streamlines in the two cases.

7. Numerical solution of the axisymmetric problem. We consider the penetration of a finger into a tube. As in the two-dimensional case, the steady state problem is examined and the finger moves parallel to the $x$-axis with constant velocity $U$. The diameter of the tube is $2 b$ and the diameter of the finger is $2 \beta b$. The parameter $\beta$ is equal to (diameter of finger)/(diameter of tube). 
(a)

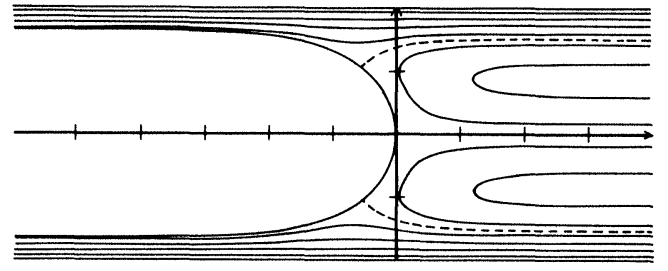

(b)

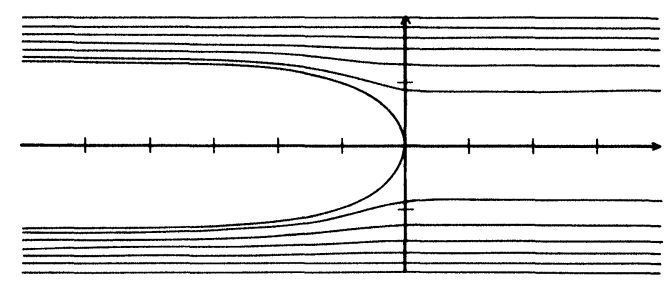

FIG. 6. Typical shape of streamlines relative to the finger. (a) $\beta>\frac{2}{3}$, two-dimensional case; $\beta>1 / \sqrt{2}$, axisymmetric case. (b) $\beta>\frac{2}{3}$, two-dimensional case; $\beta>1 / \sqrt{2}$, axisymmetric case.

The same dimensionless variables used in the two-dimensional case are used here. We write the equations in terms of the stream function $\psi$ defined by

$$
u=\frac{1}{y} \psi_{y}, \quad v=-\frac{1}{y} \psi_{x}
$$

and the vorticity $\omega$ defined by

$$
\omega=v_{x}-u_{y}
$$

The equations for $\psi$ and $\omega$ in axisymmetric Stokes flow are

$$
\begin{aligned}
& \omega_{x x}+\psi_{y y}-\frac{1}{y} \psi_{y}=-y \omega, \\
& \omega_{x x}+\omega_{y y}+\frac{1}{y} \omega_{y}-\frac{1}{y^{2}} \omega=0 .
\end{aligned}
$$

The variable $y$ is used for the radial coordinate to avoid confusion with the $r$ coordinate used in the curvilinear grid.

The interface is described by $(x(s), y(s))$ where $s$ is the arclength along the interface curve. In the axisymmetric case, the boundary conditions on the interface are given by

$$
\begin{gathered}
\psi=0, \\
y \omega-2 x_{s s} \psi_{x}-2 y_{s s} \psi_{y}=0, \\
p-2 \mathrm{Ca}\left[y_{s}^{2} u_{x}-x_{s} y_{s}\left(v_{x}+u_{y}\right)+x_{s}^{2} v_{y}\right]+\left(\frac{1}{R_{1}}+\frac{1}{R_{2}}\right)=0,
\end{gathered}
$$

where

$$
\begin{aligned}
& u_{x}=\frac{1}{y} \psi_{x y}, \quad u_{y}=\frac{1}{y} \psi_{y y}-\frac{1}{y^{2}} \psi_{y}, \\
& v_{x}=-\frac{1}{y} \psi_{x x}, \quad v_{y}=-\frac{1}{y} \psi_{x y}+\frac{1}{y^{2}} \psi_{x} .
\end{aligned}
$$


The principal curvatures for the axisymetric problem are

$$
\frac{1}{R_{1}}=x_{s} y_{s s}-y_{s} x_{s s}, \quad \frac{1}{R_{2}}=-\frac{x_{s}}{y} .
$$

The pressure $p_{0}$ is the constant pressure inside the finger and is set equal to zero. The boundary conditions on the wall of the tube are

$$
\psi(x, 1)=-\frac{1}{2}\left(1-\beta^{2}\right), \quad \psi_{y}(x, 1)=-1,
$$

and the symmetry conditions on the centerline are

$$
\psi(x, 0)=0, \quad \omega(x, 0)=0 .
$$

The asymptotic behaviours of $\psi$ and $w$ are

$$
\begin{aligned}
& \psi \rightarrow-\frac{1}{2}\left(y^{2}-\beta^{2}\right) \text { and } \omega \rightarrow 0 \text { as } x \rightarrow-\infty, \\
& \psi \rightarrow \frac{1}{2} \beta^{2}\left(2 y^{2}-y^{4}\right)-\frac{1}{2} y^{2} \text { and } \omega \rightarrow 4 \beta^{2} y \quad \text { as } x \rightarrow \infty .
\end{aligned}
$$

As in the two-dimensional case, the normal-stress boundary condition is dropped, and the numerical solution is computed on a fixed domain. The normal-stress boundary condition is used to find the shape of the finger.

The results for the axisymmetric problem are very similar to the two-dimensional results. In Fig. 7, the solid line is a plot of $\beta$ versus $\mu U / T$ calculated from the numerical

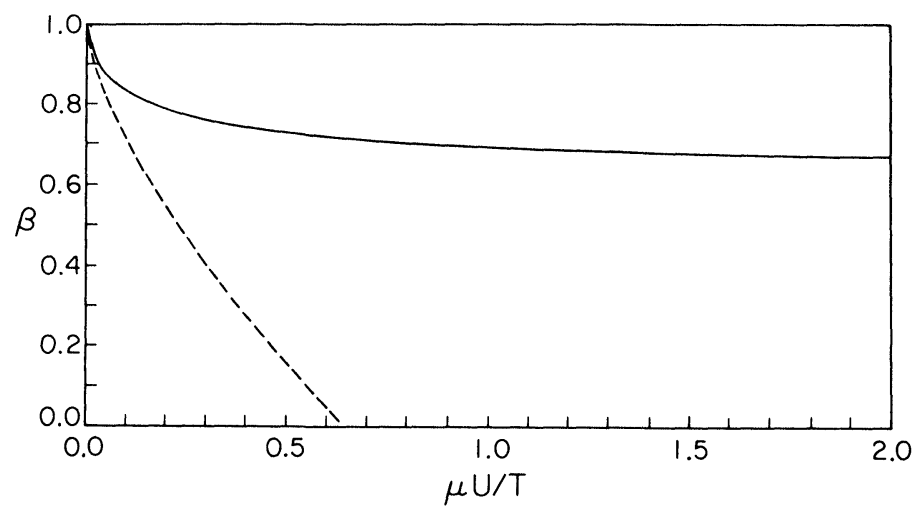

FIG. 7. The finger width $\beta$ versus $\mu U / T$ for the axisymmetric problem. - _ numerical results; -- , perturbation result (19).

solutions and the dashed line is the perturbation result (19). As $x \rightarrow \infty$, the pressure is given by

$$
p \sim-8 \beta^{2} \mathrm{Ca} x+c_{p}
$$

where $c_{p}$ is a constant. The solid line in Fig. 8 shows $\Delta p$, the pressure drop across the tip of the finger, and the dotted line gives $c_{p}$. Both $\Delta p$ and $c_{p}$ have been normalized by $T / b$.

As $x \rightarrow \infty$, the velocity in the $x$-direction is

$$
u \rightarrow 2 \beta^{2}\left(1-y^{2}\right)-1 \text {. }
$$

For $\beta$ greater than $1 / \sqrt{2}$, the fluid near the $x$-axis moves faster than the finger. Taylor (1961) discusses the two simplest types of flows that might occur: a stagnation point 


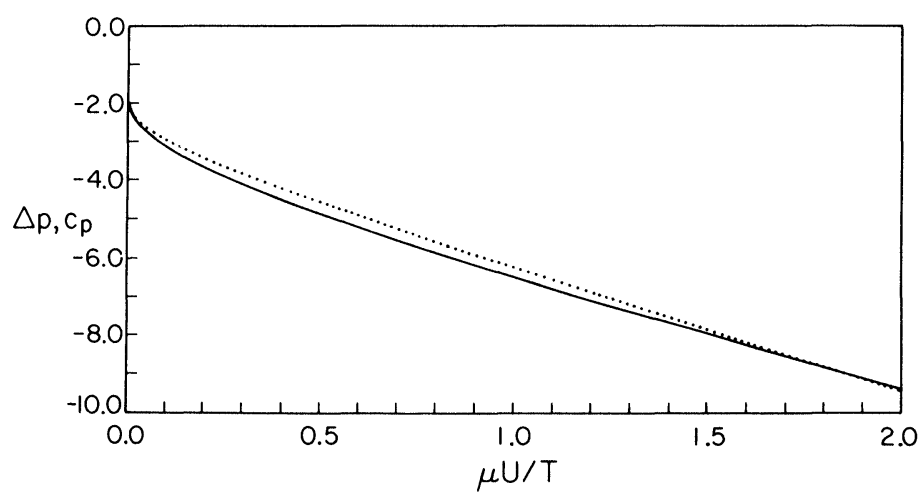

FIG. 8. - pressure drop $\Delta p$ across tip of the finger versus $\mu U / T$ for the axisymmetric problem; $\cdots$, $c_{p}$ versus $\mu U / T$.

at the origin with a stagnation ring on the interface of the finger or two stagnation points on the $x$-axis, one of which is at the origin. By examining Fig. 6, it is clear that a stagnation ring is present for $\beta$ greater than $1 / \sqrt{2}$.

When the axisymmetric finger moves through the tube, a fraction $m$ of the viscous fluid is left behind on the walls of the tube. The fraction $m$ was measured experimentally by Taylor as a function of $\mu U / T$. Figure 9 compares the numerical results with the experimental results where $m$ is equal to $1-\beta^{2}$. The numerical results are in excellent agreement with the experimental results.

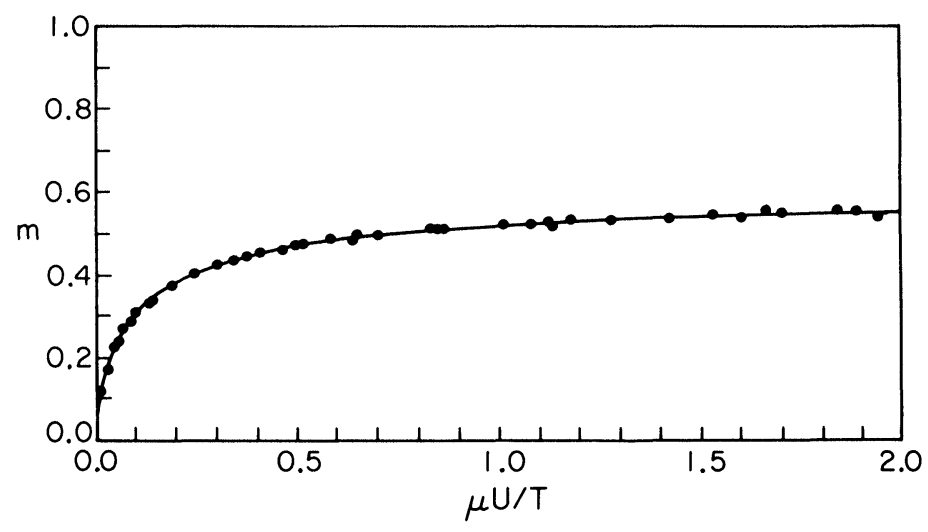

FIG. 9. Fraction $m$ of viscous fluid left behind on the walls of the tube versus $\mu U / T$. _, numerical result; •, Taylor experimental results.

8. Conclusion. In solving the fingering problem, we have used a composite mesh to cover the domain. The resulting numerical solution is not only accurate in the interior of the region but also on the boundaries of the domain. The amount of computing time necessary to construct the grids is a very small percentage of the time necessary to compute the solution to the fingering problem.

The employment of a composite mesh creates enough flexibility that it can be used to treat problems with many different types of geometries. It can also be used in determining solutions that exhibit singular behavior. The composite mesh can be composed of as many grids as necessary to solve a given problem. The grids are easily constructed to include stretching which places grid points where they are needed most. 
The numerical methods employed work very well in the treatment of this free surface problem. Many other free surface problems could be examined by extending the methods to include the effects of the inertia terms. The effects of gravity on the shape of the finger for the two-dimensional and axisymmetric geometries can also be calculated. The methods could also be extended to handle time-dependent free surface problems. In these problems, the curvilinear grid would move with the interface at each time step of the calculation.

Appendix. As discussed earlier, stretching functions are introduced to place grid points where they are needed most. In the $x$ direction, fewer grid points are needed near $x_{\min }$ and $x_{\max }$ where the solution tends to a function of $y$ only. The $x$ dependence is a decaying exponential. The function $f$ takes the form

$$
\hat{x}=f(x)=A x+B+C \cdot D \tanh \left(\frac{x-x_{0}}{D}\right)
$$

and the first derivative is

$$
\frac{d \hat{s}}{d x}=f^{\prime}(x)=A+C \operatorname{sech}^{2}\left(\frac{x-x_{0}}{D}\right) .
$$

$A$ and $C$ are chosen such that there is a larger mesh size near the boundaries and a smaller mesh size in an interior region centered about the point $x_{0}$. The constant $D$ is the decay rate from the smaller mesh size to the larger one. $B$ is chosen such that $f\left(x_{\min }\right)=0$.

In the perturbation problem, it was found that for small $\mathrm{Ca}$ the finger nearly fills the channel. To numerically solve the fingering problem for this case, it is necessary to have a small mesh size near $y=1$. The stretching in the $y$ direction takes the form

$$
\hat{y}=g(y)=A y+C \cdot D\left[\exp \left(\frac{-(1-y)}{D}\right)-\exp \left(\frac{-(1+y)}{D}\right)\right]
$$

where the first derivative is given by

$$
\frac{d \hat{y}}{d y}=g^{\prime}(y)=A+C\left[\exp \left(\frac{-(1-y)}{D}\right)+\exp \left(\frac{-(1+y)}{D}\right)\right] \text {. }
$$

The constants $A$ and $C$ are chosen to produce a small mesh size near $y=1$ and a larger one away from $y=1$. $D$ is the decay rate between the two mesh sizes.

In the $s$ direction, we use a stretching transformation that produces more grid points in the region near the tip of the finger and fewer in the region where the width approaches a constant. The transformation is given by

$$
\hat{s}=F(s)=A s+C \cdot D \tanh \left(\frac{s}{D}\right)
$$

where the derivative is

$$
\frac{d \hat{s}}{d s}=F^{\prime}(s)=A+C \operatorname{sech}^{2}\left(\frac{s}{D}\right)
$$

Again, $A$ and $C$ are chosen to produce the appropriate mesh sizes and $D$ is the decay rate. In the fingering problem it is not necessary to stretch in the $r$ direction, so we simply set $\hat{r}=G(r)=r$. 
Acknowledgments. We wish to thank Prof. H. O. Kreiss for suggesting the composite mesh method and B. Kreiss for help with the initial implementation.

\section{REFERENCES}

G. K. BATChelor (1967), An Introduction to Fluid Mechanics, Cambridge Univ. Press, London.

F. P. BRETHerton (1961), The motion of long bubbles in tubes, J. Fluid Mech., 10, pp. 166-188.

B. G. Cox (1962), On driving a viscous fluid out of a tube, J. Fluid Mech., 14, pp. 81-96.

G. Dahlquist ANd A. BJorck (1974), Numerical Methods, Prentice-Hall, Englewood Cliffs, NJ.

B. KREISS (1983), Construction of a curvilinear grid, this Journal, 4, pp. 270-279.

H. LAMB (1932), Hydrodynamics, Sixth Edition, Dover, New York.

J. W. MCLEAN AND P. G. SAFFMAN (1980), The effect of surface tension on the shape of fingers in a Hele-Shaw cell, J. Fluid Mech., 102, pp. 455-469.

C. W. PARK AND G. M. HOMSY (1983), Two-phase displacement in Hele-Shaw cells, submitted for publication.

E. PITTS (1980), Penetration of fluid into a Hele-Shaw cell: the Saffman-Taylor experiment, J. Fluid Mech., 97, pp. 53-64.

D. A. Reinelt (1983), Ph.D. dissertation, California Institute of Technology, Pasadena.

P. J. RoAChe (1976), Computational Fluid Dynamics, Hermosa, Albuquerque, NM.

L. A. Romero (1982), Ph.D. dissertation, California Institute of Technology, Pasadena.

P. G. Saffman (1982), Fingering in porous media, Lecture Notes in Physics, 154, Burridge et al., eds., Springer-Verlag, pp. 208-214.

P. G. SAFFMAN AND G. I. TAYLOR (1958), The penetration of a fluid into a porous medium or Hele-Shaw cell containing a more viscous liquid, Proc. Roy. Soc. A, 245, pp. 312-329.

G. I. TAYLOR (1961), Deposition of a viscous fluid on the wall of a tube, J. Fluid Mech., 10, pp. 161-165.

J.-M. VANDEN-BroecK (1983), Fingers in a Hele-Shaw cell with surface tension, Phys. Fluids, 26 (8), pp. 2033-2034. 